\title{
MODEL SHOWING DISTRIBUTION OF ELECTROMOTIVE FORCE AND CURRENT ALONG A SINGLE PHASE ALTERNATING CURRENT TRANSMISSION LINE.
}

BY W. S. FRANKLIN.

A helical line drawn on the surface of a transparent cylinder and set rotating about its axis would appear to an observer looking at it from one side as a wave-line traveling along the axis. The direction of travel of this wave-line would be one way or the other, according as the helix is right or left-handed.

A rotating right-handed helix would represent the electromotive force waves (or the current waves) traveling outwards from a generating station along a transmission line having inductance and capacity, but having neither resistance nor leakage.

A left-handed helix rotating in the same direction about the same axis would represent the electromotive force waves (or the current waves) reflected back from the receiver. A compound helix of which the radius-vector at each point along the axis is the sum of the radii vectores of the two simple helices would, if set rotating, represent the actual distribution of electromotive force (or current) along the transmission line.

The effect of line resistance and line leakage is to cause the waves of electromotive force and current to diminish in amplitude as they travel along the line.

Imagine a helix-like line drawn about an axis, the radiusvector of this helix-like line at a point along the axis being proportional to $\varepsilon^{-x}$ where $\varepsilon$ is the Naperian base and $x$ is distance measured along the axis from one end. If this helix-like line is set rotating about the axis. it will appear to an observer looking at it 
from one side as a wave-line traveling along the axis and diminishing in amplitude as it travels along.

A rotating, right-handed helix-like line of the kind just specified would represent the electromotive force waves (or the current waves) traveling outwards from a generating station along a transmission line having inductance, capacity, resistance and leakage.

A left-handed helix-like line rotating in the same direction about the same axis would represent the electromotive force waves (or the current waves) which are reflected back from the receiver.

A compound helix-like line of which the radius-vector at each point along the axis is the vector sum of the radii vectores of the two single helix-like lines, would, if set rotating, represent the actual distribution of electromotive force (or current) along the transmission line.

The model which is herewith exhibited contains: (A), Two simple and one compound helix-like lines, which represent outgoing, reflected, and resultant electromotive force waves respectively; and (в), Two simple and one compound helix-like lines which represent outgoing, reflected, and resultant current waves respectively.

This model was calculated and constructed by Messrs. J. H. Flory and C. W. Startsman, as a graduating thesis in electrical cngineering, at Lehigh University in 1901.

The calculations were based upon the following data:

Length of line, 200 miles.

Resistance of line one ohm per mile.

Reactance of line due to inductance four ohms per mile.

Leakage conductance of line, 0.00002 mho's per mile.

Capacity susceptance of line, 0.0002 mho's per mile.

Receiver is a condenser having a reactance value of $100 \mathrm{ohms}$.

The actual value of electromotive force (or current) is not essential to the interpretation of the model but as a matter of fact the voltage at the terminals of the receiving circuit was taken to be 10,000 volts and the current, of course, 100 amperes. 